\title{
W. von Tschirnhaus and algebraic equations with roots in arithmetical progression
}

\author{
By Gino Loria. \\ (Received and read 3rd March, 1940.) \\ Communicated by Professor H. W. Ternbull.
}

In an interesting appendix to a letter written by John Collins to James Gregory on August 3, 1675, but not published until a few months ago, appear some formulae, given without proof, for expressing the roots of an equation of any degree from the 2 nd to the 9 th in terms of the coefficients, under the assumption that these roots are in arithmetical progression. The formulae were discovered by the well known contemporary of Leibniz, Baron $W$. von Tschirnhaus. It is evident that in the case of an equation of degree $n$ this particular assumption imposes $n-2$ conditions on the coefficients; so that two of these coefficients can be chosen ad libitum. Tschirnhaus did not go to the trouble of obtaining these relations explicitly, in fact he makes no mention of them, but he gives expressions, in the cases indicated above, for the roots as functions of the first two coefficients of the equation in question, and these coefficients, as we have observed, are arbitrary. It is not known by what approach he arrived at his formulae; it seems likely to us, however, that he expressed the desired roots in terms of two arbitrary unknowns, that he evaluated the sum of these, and the sum of their products two at a time, and that, finally, he equated the results to the first two coefficients of the equation. In this way two equations are obtained, sufficient to determine the two auxiliary unknowns; and the problem can be considered as solved. Without seeming to imply that this procedure was the same as that adopted by the eminent German mathematician, we shall show that by its means one can not only derive his results, but also solve the question in the case of an algebraic equation of any degree.

To this end it is necessary to consider separately the cases of even and of odd degree.

Case 1. Let the proposed equation be

$$
x^{2 n+1}+p x^{2 n}+q x^{2 n-1}+\ldots=0 .
$$


If the mean root is $x$ then all the roots, presumed in arithmetical progression, can be represented thus,

$$
x-n y, \ldots ., x-y, x, x+y, \ldots, x+n y,
$$

and since their sum is $(2 n+1) x$, we must have $(2 n+1) x=-p$, whence

$$
x=-p /(2 n+1) .
$$

We apply next the identity

$$
2(a b+a c+b c+\ldots)=(a+b+c+\ldots)^{2}-\left(a^{2}+b^{2}+c^{2}+\ldots\right)
$$

and (3). It follows that the expression to be equated to $2 q$ is

$$
(-p)^{2}-\left\{x^{2}+(x-y)^{2}+(x+y)^{2}+\ldots+(x-n y)^{2}+(x+n y)^{2}\right\},
$$

that is,

$$
p^{2}-\left\{(2 n+1) x^{2}+2 y^{2}\left(1^{2}+2^{2}+\ldots .+n^{2}\right),\right.
$$

and this, in view of

$$
1^{2}+2^{2}+\ldots+n^{2}=n(n+1)(2 n+1) / 6,
$$

can be written

$$
p^{2}-\left\{\frac{p^{2}}{2 n+1}+2 y^{2} \frac{n(n+1)(2 n+1)}{6}\right\},
$$

so that we have

$$
2 q=\frac{2 n p^{2}}{2 n+1}-2 y^{2} \frac{n(n+1)(2 n+1)}{6} .
$$

Hence

$$
y=\frac{1}{\sqrt{\frac{n(n+1)(2 n+1)}{6}}} \sqrt{\frac{n p^{2}}{2 n+1}-q},
$$

and from (2), (3), (6) we have the following expressions for the roots,

$$
-\frac{p}{2 n+1}+\frac{i}{\sqrt{\frac{n(n+1)(2 n+1)}{6}}} \sqrt{\frac{n p^{2}}{2 n+1}-q},
$$

where $i$ takes integer values from $-n$ to $n$.

If in (7) we put in succession $n=1,2,3,4$ we obtain

$$
\sqrt{\frac{p^{2}}{3}-q}, \frac{1}{\sqrt{5}} \sqrt{\frac{2 p^{2}}{5}-q}, \frac{1}{\sqrt{14}} \sqrt{\frac{3 p^{2}}{7}}-q, \frac{1}{\sqrt{30}} \sqrt{\frac{4 p^{2}}{9}-q},
$$

expressions which lead immediately to four of the values of the roots given by Tschirnhaus. 
Case 2. Let the proposed equation be

$$
x^{2 n}+p x^{2 n-1}+q x^{2 n-2}+\ldots=0 .
$$

If the roots are in arithmetical progression they can be represented by (9) $x-(2 n-1) y, \ldots, x-3 y, x-y, x+y, x+3 y, \ldots, x+(2 n-1) y$, and since their sum is $2 n x$, we have $2 n x=-p$, whence

$$
x=-p / 2 n .
$$

Referring once again to (4) we see that the expression to be equated to $2 q$ in this case is

$$
\begin{aligned}
(-p)^{2}-\left\{(x-y)^{2}+(x+y)^{2}+(x-3 y)^{2}+(x+3 y)^{2}+\ldots\right. \\
\left.+(x-\overline{2 n-1} y)^{2}+(x+\overline{2 n-1} y)^{2}\right\},
\end{aligned}
$$

that is,

$$
p^{2}-\left\{2 n x^{2}+2 y^{2}\left(1^{2}+3^{2}+\ldots+\overline{2 n-1^{2}}\right) .\right.
$$

Now we have

$$
\begin{aligned}
1^{2}+3^{2} & +\ldots+(2 n-1)^{2} \\
& =\left[1^{2}+2^{2}+3^{2}+\ldots+(2 n)^{2}\right]-4\left[1^{2}+2^{2}+3^{2}+\ldots+n^{2}\right] \\
& =\frac{1}{6}\{2 n(2 n+1)(4 n+1)-4 n(n+1)(2 n+1)\} \\
& =(2 n-1) n(2 n+1) / 3,
\end{aligned}
$$

so that the expression becomes

which gives

$$
p^{2}-\left\{\frac{p^{2}}{2 n}+2 y^{2} \frac{(2 n-1) n(2 n+1)}{3}\right\}
$$

whence finally

$$
2 q=\frac{2 n-1}{2 n} p^{2}-2 y^{2} \frac{(2 n-1) n(2 n+1)}{3},
$$

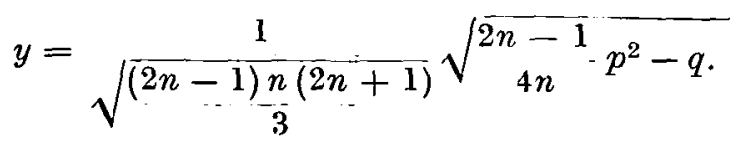

Finally, applying (8), (9) and (11), w. arrive at the following expressions for the roots,

$$
-\frac{p}{2 n} \pm \frac{2 n-i}{\sqrt{\frac{(2 n-1) n(2 n+1)}{3}}} \sqrt{\frac{2 n-1}{4 n}} p^{2}-q,
$$

where $i$ takes the values $1,2, \ldots, 2 n-1$. 
If in (11) we put in succession $n=1,2,3,4$ we find for $y$ the values

$$
\sqrt{\frac{p^{2}}{4}-q}, \frac{1}{\sqrt{10}} \sqrt{\frac{3 p^{2}}{8}-q}, \frac{1}{\sqrt{35}} \sqrt{\frac{5 p^{2}}{12}-q}, \frac{1}{\sqrt{84}} \sqrt{\frac{7 p^{2}}{16}-q} .
$$

and these expressions lead at once to the other four roots given by Tschirnhaus.

\section{NOTES}

1. See the important volume, James Gregory: Tercentenary Memorial Volume, edited by H. W. Turnbull (London, 1939), pp. 318-319.

2. In the formulae cited, the denominators $10,5,35,14,84,30$ should be replaced by their square roots.

3. The reader wishing for detailed information concerning the life and work of this mathematician will find it given at pp. 540-545 of Tome II (Milan, Hoepli, 1931) of my Storia delle Matematiche.

\section{Università DI Genova,}

ITALY. 